\title{
Adherence to optimal heart rate control in heart failure with reduced ejection fraction: insight from a survey of heart rate in heart failure in Sweden (HR-HF study)
}

\author{
M. Fu ${ }^{1,10}$ - U. Ahrenmark ${ }^{2}$ S. Berglund ${ }^{3}$ C. J. Lindholm ${ }^{4}$ - A. Lehto ${ }^{5}$. \\ A. Månsson Broberg ${ }^{6}$ - G. Tasevska-Dinevska ${ }^{7}$ G. Wikstrom ${ }^{8}$ • A. Ågard ${ }^{9}$. \\ B. Andersson ${ }^{10}$ - All investigators of the HR-HF study
}

Received: 30 March 2017 / Accepted: 31 July 2017 / Published online: 9 August 2017

(C) The Author(s) 2017. This article is an open access publication

\begin{abstract}
Introduction Despite that heart rate (HR) control is one of the guideline-recommended treatment goals for heart failure (HF) patients, implementation has been painstakingly slow. Therefore, it would be important to identify patients who have not yet achieved their target heart rates and assess possible underlying reasons as to why the target rates are not met.

Materials and methods The survey of HR in patients with HF in Sweden (HR-HF survey) is an investigator-initiated, prospective, multicenter, observational longitudinal study designed to investigate the state of the art in the control of $\mathrm{HR}$ in $\mathrm{HF}$ and to explore potential underlying mechanisms for suboptimal HR control with focus on awareness of and adherence to guidelines for HR control among physicians who focus on the contributing role of beta-blockers (BBs).
\end{abstract}

On behalf of all investigators of the HR-HF study are listed in the Acknowledgements section.

\section{Fu}

Michael.fu@gu.se; Michael.fu@vgregion.se

1 Department of Molecular and Clinical Medicine, Institute of Medicine, Sahlgrenska Academy, University of Gothenburg, Göteborg, Sweden

2 Department of Medicine, Hospital in Halmstad, Halmstad, Sweden

3 Department of Medicine, Hospital in Falun, Falun, Sweden

4 Capio City Clinic, Lund, Sweden

5 Department of Medicine, Northern Älvsborg County Hospital, Trollhättan, Sweden

6 Division of Cardiology, Department of Medicine, Huddinge, Karolinska Institutet, Karolinska University Hospital Stockholm, Stockholm, Sweden
Results In 734 HF patients the mean HR was $68 \pm 12$ beats per minute (bpm) (37.2\% of the patients had a HR $>70 \mathrm{bpm}$ ). Patients with HF with reduced ejection fraction (HFrEF) $(n=425)$ had the highest HR $(70 \pm 13 \mathrm{bpm}$, with $42 \%$ $>70 \mathrm{bpm}$ ), followed by HF with preserved ejection fraction and HF with mid-range ejection fraction. Atrial fibrillation, irrespective of HF type, had higher HR than sinus rhythm. A similar pattern was observed with BB treatment. Moreover, non-achievement of the recommended target HR ( $<70 \mathrm{bpm})$ in HFrEF and sinus rhythm was unrelated to age, sex, cardiovascular risk factors, cardiovascular diseases, and comorbidities, but was related to $\mathrm{EF}$ and the clinical decision of the physician. Approximately $50 \%$ of the physicians considered a HR of $>70 \mathrm{bpm}$ optimal and an equal number considered a HR of $>70 \mathrm{bpm}$ too high, but without recommending further action. Furthermore, suboptimal HR control cannot be attributed to the use of BBs because there was neither a difference in use of BBs nor an interaction with BBs for HR $>70 \mathrm{bpm}$ compared with HR $<70 \mathrm{bpm}$.

7 Department of Cardiology, Malmö University Hospital, University of Lund, Malmö, Sweden

8 Department of Cardiology, Academic University Hospital, Uppsala University, Uppsala, Sweden

9 Department of Medicine, Angered Hospital, Göteborg, Sweden

10 Section of Cardiology, Department of Medicine, Sahlgrenska University Hospital/Östra Hospital, 41650 Göteborg, Sweden 
Conclusion Suboptimal control of HR was noted in HFrEF with sinus rhythm, which appeared to be attributable to physician decision making rather than to the use of BBs. Therefore, our results underline the need for greater attention to HR control in patients with HFrEF and sinus rhythm and thus a potential for improved HF care.

Keywords Heart rate $\cdot$ Heart failure $\cdot$ Awareness . Adherence $\cdot$ Beta-blocker

\section{Introduction}

Available international guidelines for heart failure (HF) with reduced ejection fraction (HFrEF) recommend the following pharmacological therapies: angiotensin-converting enzyme inhibitors (ACEIs) or angiotensin receptor blockers (ARBs) if the patient is intolerant to ACEIs, beta-blockers (BBs), mineralocorticoid receptor antagonists (MRAs), ivabradine and sacubitril-valsartan [1-3]. Although the implementation of clinical guidelines generally takes time, we have witnessed a gradual improvement and increased adherence to treatment with ACEIs/ARBs, BBs, and MRAs across different countries in the past two decades [4-8]. For example, the prescription of BBs has increased in Europe from $37 \%$ in 2000 to $87-91 \%$ today [6-8]. However, for newer drugs, such as ivabradine, implementation has been slower. For instance, Dierckx et al. reported that of patients with HFrEF, 94\% were treated with BBs and only $4 \%$ were taking ivabradine [9]. One possible reason is physician-related factors, such as lack of awareness of and/or adherence to optimal heart rate (HR) control as part of the treatment goal in HFrEF and sinus rhythm. Lack of adherence has previously been suggested as one contributing factor for suboptimal HF care [10-13]. Another reason is assumed to be due to differences in use of BBs between Sweden and other countries. BBs are frequently used in the treatment of HFrEF in Sweden and could, therefore, contribute to better HR control and hence decrease the indication for further HR reduction with ivabradine. At present, while prescriptions of BBs are largely similar between Sweden and rest of the world [7, 14], the mean doses of BBs were higher in Sweden than those in other countries [6-10,14]. According to the Swedish Heart Failure Registry (SwedeHF, $n=69,527$, mean age 75 years), $67 \%$ of the patients with HFrEF were treated with BBs at $\geq 50 \%$ of the target doses. Among those $<65$ years, $77 \%$ of male and $68 \%$ of female patients were at $\geq 50 \%$ of the target doses [14]. However, according to the QUALIFY global registry, only $52 \%$ of HFrEF patients (mean age 63 years) were treated with BBs in $\geq 50 \%$ of the target doses [9]. Therefore, lower use of ivabradine in Sweden was assumed to be related to the more effective use of BBs.
The survey of HR in patients with HF in Sweden (HRHF) was an investigator-initiated, prospective, multicenter, observational longitudinal study designed to investigate the status of HR control in an outpatient cohort of stable patients with HFrEF compared with patients with HF and mid-range ejection fraction (HFmrEF) and HF with preserved ejection fraction (HFpEF) in both sinus rhythm and AF. Moreover, we explored underlying reasons to suboptimal HR control.

The main objective of the study was to assess awareness of an adherence to HR control among physicians, particularly as it contributed to the use of BBs (prescription and doses). We hypothesized that a substantial proportion of patients would have HRs above 70-75 bpm.

\section{Materials and methods}

\section{Protocol of the HR-HF study}

The HR-HF study was a prospective, multicenter, observational longitudinal survey of HF outpatients that included 734 patients in 27 centers in Sweden. These centers were hospital HF outpatient clinics with either dedicated HF nurse specialists or general practitioners. Eligible patients were those with established HF in an outpatient setting and considered on stable HF medication regimens.

The survey was carried out from 2014 to 2016 with a planned follow-up from 2017. The following variables were recorded as baseline data: demographics, diagnostic validation with left ventricular ejection fraction (LVEF), $\mathrm{N}$-terminal pro-b-type natriuretic peptide (NT-pro-BNP) or B-type natriuretic peptide (BNP), hospitalizations due to $\mathrm{HF}$ in the past 2 years, cardiovascular risk factors, cardiovascular diseases, non-cardiovascular diseases, symptoms (breathlessness, tiredness and chest pain, Likert scale), blood pressure (sitting, standing, lying), HF and rhythm (by ECG), New York Heart Association (NYHA) functional class, ADL (activity of daily living), use of BBs (up-titration, $\geq 50 \%$ of the target dose, target dose or above target dose, reasons for not being on BB treatment, reasons for not achieving target dose, side effects), use of ACEIs/ARBs/MRAs (up-titration, dose, reasons for not on treatment, reasons for not achieving target dose, side effects), other pharmacologic treatments, cardiac resynchronization therapy (CRT) device, implantable cardioverter defibrillator (ICD) device, and physicians' judgment regarding actual HR.

Different from most available HF registries [6-8, 14], the HR-HF survey focused on stable HF patients and only in outpatient settings with a special interest in HF control. Further, there was a dedicated focus on collecting information that might influence HF, for example, comorbidities and their gradings, symptoms and gradings, blood pressure, 
medications (prescriptions, dose, tolerability, side effects), and clinical judgment in relation to HF.

This study adhered to the guidelines available for human studies, including an approved ethical permit, which complies with the Helsinki Declaration and the International Ethical Guidelines for Good Clinical Practice. The study was approved by the Regional Ethical Review Board at the University of Gothenburg.

\section{Study population}

Patients eligible for entry into the survey were outpatient adults ( $>18$ years old) with a well-established diagnosis of HF based on the latest European Society of Cardiology guidelines $[1,3]$ and according to the responsible investigator's clinical judgment; an abnormal echocardiography investigation that was congruent with the HF diagnosis; optimal treatment (physicians decision) and are, therefore, not planned for further up-titration; and a stable HF condition and plans for further outpatient follow-up. The LVEF cutoffs used to define HFrEF, HFmrEF, and HFpEF were $<40,40-49$, and $\geq 50 \%$, respectively. No exclusion criteria were applied, except for those who did not or could not provide informed consent.

\section{Baseline evaluation and data management}

Data were collected centrally using a case report form that was sent to the data management center, where checks for completeness, internal consistency, and accuracy were run. Forty-nine patients were excluded from the database because of protocol deviations or incompleteness.

\section{Statistical analysis}

For categorical variables, $n(\%)$ was presented. For continuous variables, mean (SD)/median (Min/Max/n) was presented. For comparison between the three EF groups, the Mantel-Haenszel Chi-square statistic was used for ordered categorical variables, the Chi-square test for non-ordered categorical variables, and the Jonckheere-Terpstra test for continuous variables. For comparison between groups in different HRs, Fisher's exact test (lowest one-sided $p$ value multiplied by 2) was used for dichotomous variables, the Mantel-Haenszel Chi-square test for ordered categorical variables, and the Mann-Whitney $U$ test for continuous variables. For interaction and subgroup analyses in reaching a HR $>70 \mathrm{bpm}$, logistic regression was performed and odds ratios (ORs) with associated 95\% confidence intervals (CIs) and $p$ values are presented from these analyses.

All tests were two-tailed and $p$ values $<0.05$ were considered significant. All analyses were performed using SAS software version 9.4 (Cary, NC, USA).

\section{Results}

\section{Patient characteristics in the overall cohort}

Patient demographics, cardiovascular risk factors, cardiovascular diseases, non-cardiovascular diseases, clinical status, medications, and clinical assessment are outlined in Tables 1 and 2. Briefly, despite that patients with HFrEF were more often male, had more ischemic heart disease, higher NTpro-BNP, more ventricular extrasystolic couplets (VECs)/ ventricular tachycardia (VT), lower blood pressure, and more left bundle branch block (LBBB), they had a similar number of non-cardiovascular co-morbidities compared with HFmrEF and HFpEF.

\section{Medications in the overall cohort}

There were no differences in the use of BBs and ACEIs/ ARBs between the groups of HF patients, regardless of EF, with $94-97 \%$ of the patients on treatment with BBs and 93-97\% on treatment with ACEIs/ARBs (Table 2). However, in patients with HFrEF more patients were treated with MRAs, diuretics, statins, and therapy devices (CRT, ICD). In addition, patients with HFrEF were well treated with BBs (97\%), ACEIs/ARBs (97\%), MRAs (61\%), CRT (20\%), ICD (25) $9 \%$, whereas only $2.8 \%$ had ivabradine.

Concerning doses of BBs, these were similar in $\mathrm{HFrEF}$, HFmrEF, and HFpEF. Percentage of achieved target dose $\geq 50 \%$ was $79 \%$ for $\mathrm{HFrEF}, 75 \%$ for $\mathrm{HFmrEF}$, and $85 \%$ for HFpEF. For reached target dose, it was $43 \%$ for HFrEF, $45 \%$ for $\mathrm{HFmrEF}$, and $44 \%$ for $\mathrm{HFpEF}$. Moreover, $6 \%$ (HFrEF), $5 \%$ HFmrEF, and 5\% (HFpEF) of the patients had a dose above the target dose.

The main reasons why patients with HFrEF were not on treatment with BBs (3\%) were low blood pressure (22.6\%), bradycardia (15.9\%), fatigue $(9.6 \%)$, and dizziness $(9.6 \%)$. Despite that, about $97 \%$ of the patients were on treatment with BBs (only $60.6 \%$ did not report side effects). The most frequently reported side effects were tiredness $(20 \%)$, cold extremities (8.8\%), impotence (8.3\%), nightmares (3.2\%), and depression (3.2\%).

\section{Distribution of HR in the overall cohort}

In the total cohort HR was $68.4 \pm 12$ bpm with $37.2 \%$ of the patients having a HR $>70 \mathrm{bpm}$ and $22.2 \%<60 \mathrm{bpm}$ (Table 2). Patients with HFrEF presented the highest HR $(69.8 \pm 13 \mathrm{bpm}): 41.9 \%>70 \mathrm{bpm}$ and $\operatorname{HFpEF}(68.1 \pm 12)$ : $33.6 \%>70 \mathrm{bpm}$. Patients with HFmrEF had the lowest HR $(65.5 \pm 11)$, in which $28.9 \%$ had $>70$ bpm (Table 2 ; Fig. 1$)$. On average, atrial fibrillation (AF), irrespective of HFrEF, HFmrEF, and HFpEF, had a higher HR and more than $40 \%$ of the patients had a HR $>70 \mathrm{bpm}$ as compared with sinus 
Table 1 Baseline data for demographics, risk factors, and medical histories

\begin{tabular}{|c|c|c|c|c|c|}
\hline Variable & Total $(n=734)$ & $\operatorname{HFrEF}(n=425)$ & $\operatorname{HFmrEF}(n=187)$ & $\operatorname{HFpEF}(n=122)$ & $p$ value \\
\hline \multicolumn{6}{|l|}{ Demographics } \\
\hline Age (years) & $\begin{array}{l}69.1(11.6) \\
70.6(19.0 ; 95.3)\end{array}$ & $\begin{array}{l}69.8(11.2) \\
71.6(19.0 ; 95.3)\end{array}$ & $\begin{array}{l}67.8(12.3) \\
69.8(20.8 ; 89.8)\end{array}$ & $\begin{array}{l}68.7(11.8) \\
69.2(30.0 ; 89.7)\end{array}$ & 0.11 \\
\hline Male & $549(74.8 \%)$ & $337(79.3 \%)$ & $133(71.1 \%)$ & $79(64.8 \%)$ & 0.0004 \\
\hline \multicolumn{6}{|l|}{ Cardiovascular risk factors } \\
\hline Hypertension & $388(52.9 \%)$ & $213(50.1 \%)$ & $92(49.2 \%)$ & $83(68.0 \%)$ & 0.0033 \\
\hline $\mathrm{BMI}>30 \mathrm{~kg} / \mathrm{m}^{2}$ & $209(28.5 \%)$ & $121(28.5 \%)$ & $48(25.7 \%)$ & $40(32.8 \%)$ & 0.57 \\
\hline Diabetes & $181(24.7 \%)$ & $112(26.4 \%)$ & $33(17.6 \%)$ & $36(29.5 \%)$ & 0.88 \\
\hline Hypercholesterolemia & $258(35.3 \%)$ & $164(38.9 \%)$ & $56(29.9 \%)$ & $38(31.4 \%)$ & 0.045 \\
\hline Stress & $179(24.5 \%)$ & $98(23.1 \%)$ & $53(28.5 \%)$ & $28(23.0 \%)$ & 0.66 \\
\hline \multicolumn{6}{|l|}{ Cardiovascular diseases } \\
\hline Ischemic heart disease & $339(46.2 \%)$ & $218(51.3 \%)$ & $82(43.9 \%)$ & $39(32.0 \%)$ & 0.0001 \\
\hline Primary valvular disease & $89(12.1 \%)$ & $46(10.8 \%)$ & $19(10.2 \%)$ & $24(19.7 \%)$ & 0.028 \\
\hline Cardiomyopathy & $243(33.1 \%)$ & $152(35.8 \%)$ & $57(30.5 \%)$ & $34(27.9 \%)$ & 0.067 \\
\hline Chronic persistent atrial fibrillation & $201(27.4 \%)$ & $120(28.2 \%)$ & $42(22.5 \%)$ & $39(32.0 \%)$ & 0.83 \\
\hline Paroxysmal atrial fibrillation & $119(16.2 \%)$ & $68(16.0 \%)$ & $24(12.8 \%)$ & $27(22.1 \%)$ & 0.28 \\
\hline VES/VT & $130(17.7 \%)$ & $84(19.8 \%)$ & $34(18.2 \%)$ & $12(9.8 \%)$ & 0.019 \\
\hline \multicolumn{6}{|l|}{ Non-cardiovascular diseases } \\
\hline Mild/moderate pulmonary disease & $70(9.5 \%)$ & $43(10.1 \%)$ & $15(8.0 \%)$ & $12(9.8 \%)$ & 0.73 \\
\hline Severe pulmonary disease & $13(1.8 \%)$ & $7(1.6 \%)$ & $3(1.6 \%)$ & $3(2.5 \%)$ & 0.61 \\
\hline GFR $<30 \mathrm{ml} / \mathrm{min}$ & $34(4.7 \%)$ & $21(5.0 \%)$ & $8(4.3 \%)$ & $5(4.1 \%)$ & \\
\hline $30-60 \mathrm{ml} / \mathrm{min}$ & $257(35.3 \%)$ & $163(38.6 \%)$ & $51(27.6 \%)$ & $43(35.5 \%)$ & \\
\hline$>60 \mathrm{ml} / \mathrm{min}$ & $437(60.0 \%)$ & $238(56.4 \%)$ & $126(68.1 \%)$ & $73(60.3 \%)$ & 0.14 \\
\hline Stroke without sequelae & $62(8.4 \%)$ & $33(7.8 \%)$ & $17(9.1 \%)$ & $12(9.8 \%)$ & 0.42 \\
\hline Stroke with sequelae & $23(3.1 \%)$ & $15(3.5 \%)$ & $5(2.7 \%)$ & $3(2.5 \%)$ & 0.48 \\
\hline \multicolumn{6}{|l|}{ Hemoglobin (g/L) (cat.) } \\
\hline$<90$ & $4(0.6 \%)$ & $2(0.5 \%)$ & $2(1.2 \%)$ & $0(0.0 \%)$ & \\
\hline 90 to $<110$ & $29(4.5 \%)$ & $22(6.0 \%)$ & $3(1.8 \%)$ & $4(3.6 \%)$ & \\
\hline$\geq 110$ & $611(94.9 \%)$ & $342(93.4 \%)$ & $161(97.0 \%)$ & $108(96.4 \%)$ & 0.14 \\
\hline Depression & $81(11.0 \%)$ & $47(11.1 \%)$ & $20(10.7 \%)$ & $14(11.5 \%)$ & 0.95 \\
\hline Impotence & $140(29.3 \%)$ & $93(32.0 \%)$ & $35(28.2 \%)$ & $12(19.0 \%)$ & 0.046 \\
\hline Malignancy (active) & $15(2.0 \%)$ & $9(2.1 \%)$ & $4(2.1 \%)$ & $2(1.6 \%)$ & 0.78 \\
\hline Malignancy (stable) & $73(9.9 \%)$ & $45(10.6 \%)$ & $13(7.0 \%)$ & $15(12.3 \%)$ & 0.98 \\
\hline Malnutrition & $24(3.3 \%)$ & $16(3.8 \%)$ & $5(2.7 \%)$ & $3(2.5 \%)$ & 0.40 \\
\hline Liver failure & $6(0.8 \%)$ & $3(0.7 \%)$ & $1(0.5 \%)$ & $2(1.6 \%)$ & 0.42 \\
\hline Thyroid disease & $60(8.2 \%)$ & $28(6.6 \%)$ & $17(9.1 \%)$ & $15(12.3 \%)$ & 0.037 \\
\hline Gout & $97(13.2 \%)$ & $64(15.1 \%)$ & $15(8.0 \%)$ & $18(14.8 \%)$ & 0.39 \\
\hline Dementia & $3(0.4 \%)$ & $2(0.5 \%)$ & $1(0.5 \%)$ & $0(0.0 \%)$ & 0.56 \\
\hline Other important non-cardiovascular disease & $65(8.9 \%)$ & $32(7.5 \%)$ & $14(7.5 \%)$ & $19(15.6 \%)$ & 0.018 \\
\hline
\end{tabular}

rhythm (about $30 \%$ of the patients had a HR $>70 \mathrm{bpm}$ ). A similar pattern was seen in HFrEF in which about $50 \%$ of those suffering from AF had a HR $>70 \mathrm{bpm}$, whereas $34 \%$ of those with sinus rhythm had a HR $>70 \mathrm{bpm}$. The pattern of HR remained similar between sinus rhythm and AF in HFrEF despite treatment with BBs (Fig. 2).

\section{Clinical assessment by physician in the overall cohort}

Despite that $37 \%$ of all HF and $42 \%$ of all HFrEF had a HR $>70 \mathrm{bpm}, 75 \%$ of the physicians felt that the patients had optimal HR control, whereas $20 \%$ considered the patients to have a HR that was too high. 
Table 2 Baseline data for clinical status, medication, and clinical assessment by physicians

\begin{tabular}{|c|c|c|c|c|c|}
\hline Variable & Total $(n=734)$ & $\operatorname{HFrEF}(n=425)$ & $\operatorname{HFmrEF}(n=187)$ & $\operatorname{HFpEF}(n=122)$ & $p$ value \\
\hline \multicolumn{6}{|l|}{ Clinical status } \\
\hline LVEF (\%) & $\begin{array}{l}36.9(17.9) \\
35.0(10.0 ; 401.0) \\
n=734\end{array}$ & $\begin{array}{l}28.2(6.8) \\
30.0(10.0 ; 39.0) \\
n=425\end{array}$ & $\begin{array}{l}43.0(2.8) \\
42.5(40.0 ; 49.0) \\
n=187\end{array}$ & $\begin{array}{l}57.7(31.7) \\
55.0(50.0 ; 401.0) \\
n=122\end{array}$ & $<.0001$ \\
\hline NT-pro-BNP (ng/L) & $\begin{array}{l}2810(5044) \\
1251(10 ; 70,000) \\
n=629\end{array}$ & $\begin{array}{l}3255(5345) \\
1559(10 ; 70,000) \\
n=364\end{array}$ & $\begin{array}{l}2021(3936) \\
808(37 ; 30,000) \\
n=156\end{array}$ & $\begin{array}{l}2456(5291) \\
706(43 ; 35,000) \\
n=109\end{array}$ & $<.0001$ \\
\hline Sitting systolic blood pressure $(\mathrm{mmHg})$ & $\begin{array}{l}126.2(58.2) \\
120.0(54.0 ; 1500.0) \\
n=619\end{array}$ & $\begin{array}{l}121.8(17.8) \\
120.0(54.0 ; 190.0) \\
n=341\end{array}$ & $\begin{array}{l}126.1(18.0) \\
126.0(85.0 ; 180.0) \\
n=170\end{array}$ & $\begin{array}{l}140.2(133.4) \\
126.5(85.0 ; 1500.0) \\
n=108\end{array}$ & 0.0010 \\
\hline Heart rate $(\mathrm{bpm})$ by ECG & $\begin{array}{l}68.4(12.4) \\
67.0(34.0 ; 123.0) \\
n=734\end{array}$ & $\begin{array}{l}69.8(13.0) \\
68.0(34.0 ; 123.0) \\
n=425\end{array}$ & $\begin{array}{l}65.5(10.7) \\
64.0(43.0 ; 95.0) \\
n=187\end{array}$ & $\begin{array}{l}68.1(12.0) \\
66.0(44.0 ; 103.0) \\
n=122\end{array}$ & 0.0062 \\
\hline$<60$ bpm & $163(22.2 \%)$ & $81(19.1 \%)$ & $56(29.9 \%)$ & $26(21.3 \%)$ & \\
\hline $60-70 \mathrm{bpm}$ & $298(40.6 \%)$ & $166(39.1 \%)$ & $77(41.2 \%)$ & $55(45.1 \%)$ & \\
\hline$>70 \mathrm{bpm}$ & $273(37.2 \%)$ & $178(41.9 \%)$ & $54(28.9 \%)$ & $41(33.6 \%)$ & 0.019 \\
\hline LBBB & $163(22.2 \%)$ & $111(26.2 \%)$ & $37(19.8 \%)$ & $15(12.3 \%)$ & 0.0007 \\
\hline $\begin{array}{l}\text { Sinus rhythm (and not previously detected } \\
\text { persistent or paroxysmal atrial fibrillation) }\end{array}$ & $387(52.8 \%)$ & $216(50.9 \%)$ & $115(61.5 \%)$ & $56(45.9 \%)$ & 0.96 \\
\hline $\begin{array}{l}\text { Atrial fibrillation (or previously detected } \\
\text { persistent or paroxysmal) }\end{array}$ & $322(43.9 \%)$ & $191(45.0 \%)$ & $66(35.3 \%)$ & $65(53.3 \%)$ & 0.51 \\
\hline Chamber pacing & $149(20.3 \%)$ & $105(24.8 \%)$ & $26(13.9 \%)$ & $18(14.8 \%)$ & 0.0020 \\
\hline \multicolumn{6}{|l|}{ NYHA (cat.) } \\
\hline $\mathrm{I}-\mathrm{II}$ & $538(73.3 \%)$ & $301(70.8 \%)$ & $150(80.2 \%)$ & $87(71.3 \%)$ & \\
\hline III-IV & $196(26.7 \%)$ & $124(29.2 \%)$ & $37(19.8 \%)$ & $35(28.7 \%)$ & 0.37 \\
\hline \multicolumn{6}{|l|}{ Medication } \\
\hline Beta-blockers & $705(96.0 \%)$ & $411(96.7 \%)$ & $176(94.1 \%)$ & $118(96.7 \%)$ & 0.62 \\
\hline RAAS (ACEI/ARB) & $707(96.3 \%)$ & $413(97.2 \%)$ & $180(96.3 \%)$ & $114(93.4 \%)$ & 0.065 \\
\hline MRA & $407(55.4 \%)$ & $257(60.5 \%)$ & $93(49.7 \%)$ & $57(46.7 \%)$ & 0.0017 \\
\hline Loop diuretics & $420(57.2 \%)$ & $267(62.8 \%)$ & $84(44.9 \%)$ & $69(56.6 \%)$ & 0.015 \\
\hline Digitalis & $96(13.1 \%)$ & $61(14.4 \%)$ & $14(7.5 \%)$ & $21(17.2 \%)$ & 0.96 \\
\hline Statin & $417(56.8 \%)$ & $260(61.2 \%)$ & $97(51.9 \%)$ & $60(49.2 \%)$ & 0.0062 \\
\hline Ivabradine/procoralan & $21(2.9 \%)$ & $12(2.8 \%)$ & $8(4.3 \%)$ & $1(0.8 \%)$ & 0.50 \\
\hline \multicolumn{6}{|l|}{ Device treatments } \\
\hline Conventional pacemaker & $63(8.6 \%)$ & $29(6.8 \%)$ & $16(8.6 \%)$ & $18(14.8 \%)$ & 0.0091 \\
\hline CRT & $106(14.4 \%)$ & $85(20.0 \%)$ & $15(8.0 \%)$ & $6(4.9 \%)$ & $<.0001$ \\
\hline ICD & $140(19.1 \%)$ & $110(25.9 \%)$ & $19(10.2 \%)$ & $11(9.0 \%)$ & $<.0001$ \\
\hline \multicolumn{6}{|l|}{ Clinical assessment } \\
\hline $\begin{array}{l}\text { Physician considers patient having too low } \\
\text { heart rate }\end{array}$ & $21(2.9 \%)$ & $12(2.8 \%)$ & $6(3.2 \%)$ & $3(2.5 \%)$ & 0.92 \\
\hline $\begin{array}{l}\text { Physician considers patient having optimal } \\
\text { heart rate }\end{array}$ & $568(77.4 \%)$ & $320(75.3 \%)$ & $152(81.3 \%)$ & $96(78.7 \%)$ & 0.22 \\
\hline $\begin{array}{l}\text { Physician considers patient having too high } \\
\text { heart rate }\end{array}$ & $145(19.8 \%)$ & $93(21.9 \%)$ & $29(15.5 \%)$ & $23(18.9 \%)$ & 0.22 \\
\hline
\end{tabular}

\section{HR and influencing factors in HFrEF with sinus rhythm}

In HFrEF patients with sinus rhythm $33.6 \%$ had a HR $>70$ bpm. As shown in Tables 3 and 4, when all variables (demographic variables, cardiovascular risk factors, cardiovascular diseases, non-cardiovascular diseases, clinical status, medications, and clinical assessment by physicians) were compared between HR $<70 \mathrm{bpm}$ and $>70 \mathrm{bpm}$, only a few of these variables were statistically significant: EF, symptoms of breathlessness and chest pain, and physicians' clinical assessment, i.e., those HFrEF patients with HR $>70 \mathrm{bpm}$ had lower EF, were more symptomatic, and that $49 \%$ of the physicians considered a HR $>70 \mathrm{bpm}$ optimal, 


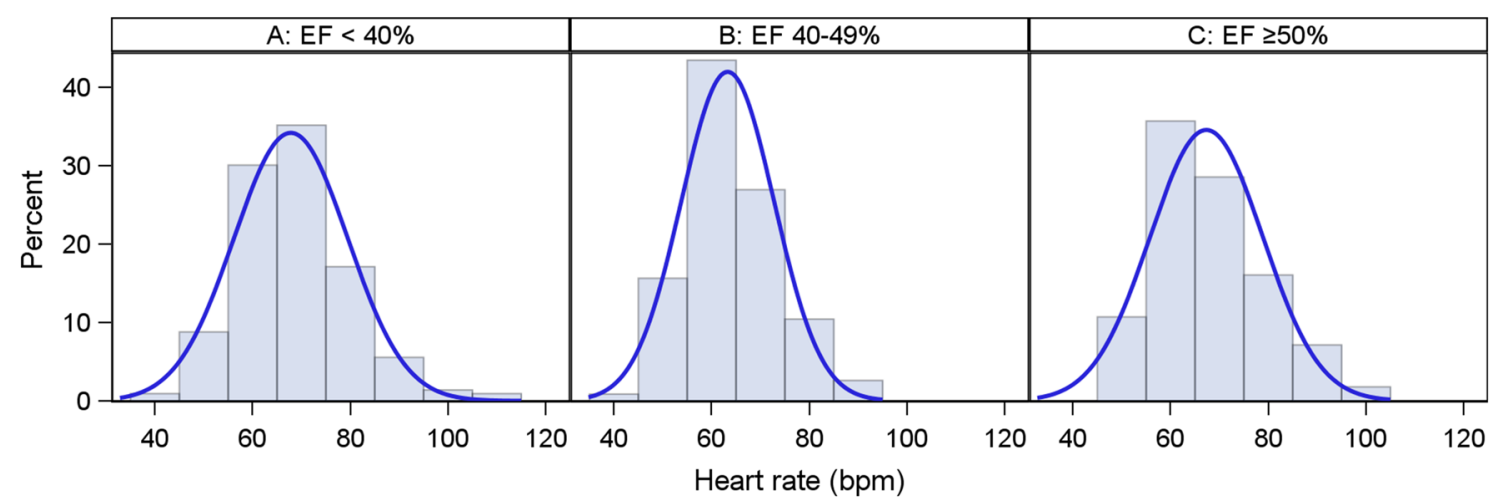

Fig. 1 Distribution of heart rate for patients with sinus rhythm and $\mathrm{EF}<40 \%$ (a), EF 40-49\% (b), and EF $\geq 50 \%$ (c)

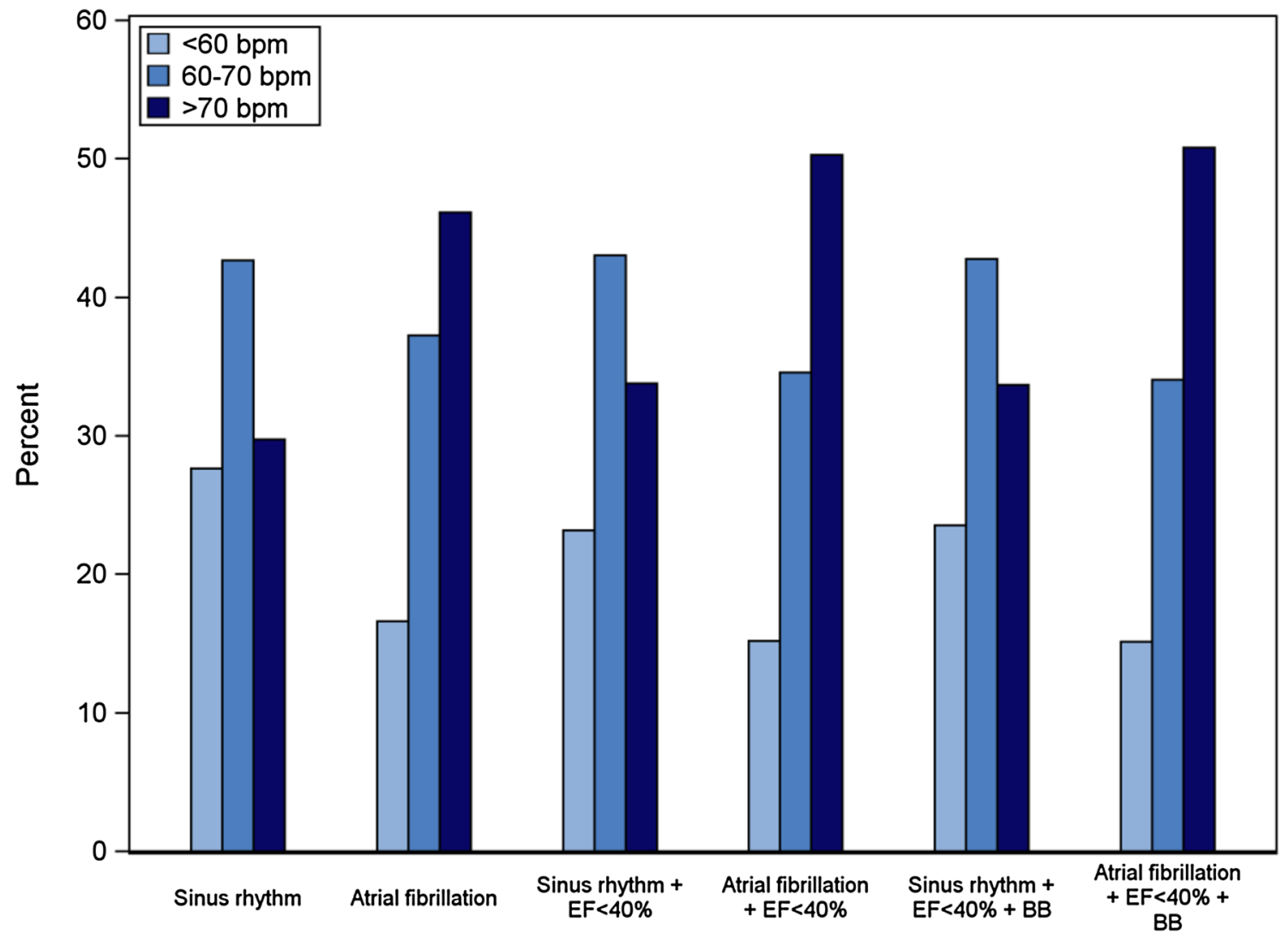

Fig. 2 Distribution of heart rate in patients with $\mathrm{EF}<40 \%$

whereas an equal number of physicians felt that a HR $>70 \mathrm{bpm}$ was too high (but without further action) (Table 4).

\section{Use of BBs in HFrEF with sinus rhythm}

As can be seen in Table 4, there were no differences between a HR $<70 \mathrm{bpm}$ and a HR $>70 \mathrm{bpm}$ in the use of $\mathrm{BBs}$, regardless of prescription, type of BBs, duration of BB use, site for BB up-titration, or dose. In HFrEF with sinus rhythm, BBs were used in $97 \%$ (HR $<70 \mathrm{bpm}$ ) and
96\% (HR $>70 \mathrm{bpm}$ ) in overall population, and in $73 \%$ (HR $<70 \mathrm{bpm}$ ) and $80 \%$ (HR $>70 \mathrm{bpm}$ ) at $\geq 50 \%$ of target dose, $38 \%$ (HR $<70 \mathrm{bpm}$ ) and $40 \%$ (HR $>70 \mathrm{bpm}$ ) at target dose, and $2 \%(\mathrm{HR}<70 \mathrm{bpm})$ and $0 \%(\mathrm{HR}>70 \mathrm{bpm})$ at a dose above target dose (Table 4).

\section{Interaction analysis}

Because the current study was aimed to explore possible contributing factors to a HR $>70 \mathrm{bpm}$ in HFrEF with sinus 
Table 3 Comparison of demographics, risk factors, and medical histories for $\mathrm{HR} \leq 70$ vs. $>70 \mathrm{bpm}$ in all patients with sinus rhythm and $\mathrm{EF}<40 \%$ (HFrEF)

\begin{tabular}{|c|c|c|c|}
\hline Variable & $\leq 70 \mathrm{bpm}(n=143)$ & $>70 \mathrm{bpm}(n=73)$ & $p$ value \\
\hline Age (years) & $\begin{array}{l}67.5(11.9) \\
68.8(25.5 ; 95.3) \\
n=143\end{array}$ & $\begin{array}{l}63.9(14.2) \\
65.8(19.0 ; 91.0) \\
n=73\end{array}$ & 0.068 \\
\hline Male & $107(74.8 \%)$ & $57(78.1 \%)$ & 0.72 \\
\hline $\operatorname{LVEF}(\%)$ & $\begin{array}{l}29.1(6.4) \\
30.0(10.0 ; 39.0) \\
n=143\end{array}$ & $\begin{array}{l}25.6(7.7) \\
25.0(10.0 ; 38.0) \\
n=73\end{array}$ & 0.0016 \\
\hline NT-pro-BNP (ng/L) & $\begin{array}{l}2451(3429) \\
1360(14 ; 25,600) \\
n=118\end{array}$ & $\begin{array}{l}3101(4981) \\
1239(81 ; 27,362) \\
n=66\end{array}$ & 0.94 \\
\hline Hemoglobin $(\mathrm{g} / \mathrm{L})$ & $\begin{array}{l}137.4(16.1) \\
139.0(86.0 ; 175.0) \\
n=123\end{array}$ & $\begin{array}{l}137.9(23.7) \\
140.0(4.0 ; 178.0) \\
n=63\end{array}$ & 0.56 \\
\hline $\begin{array}{l}\text { Number of hospitalizations due to heart failure } \\
\text { in the past } 2 \text { years }\end{array}$ & $\begin{array}{l}0.427(0.622) \\
0.000(0.000 ; 3.000) \\
n=143\end{array}$ & $\begin{array}{l}0.616(0.860) \\
0.000(0.000 ; 3.000) \\
n=73\end{array}$ & 0.18 \\
\hline \multicolumn{4}{|l|}{ Cardiovascular risk factors } \\
\hline Hypertension & $69(48.3 \%)$ & $30(41.1 \%)$ & 0.39 \\
\hline $\mathrm{BMI}>30 \mathrm{~kg} / \mathrm{m} 2$ & $38(26.6 \%)$ & $28(38.4 \%)$ & 0.11 \\
\hline \multicolumn{4}{|l|}{ Smoking } \\
\hline Never smoked & $62(43.4 \%)$ & $27(37.0 \%)$ & \\
\hline Stopped smoking & $58(40.6 \%)$ & $36(49.3 \%)$ & \\
\hline Smoking & $23(16.1 \%)$ & $10(13.7 \%)$ & 0.70 \\
\hline Diabetes & $36(25.2 \%)$ & $21(28.8 \%)$ & 0.68 \\
\hline \multicolumn{4}{|l|}{ Alcohol } \\
\hline Normal consumption & $115(93.5 \%)$ & $56(96.6 \%)$ & \\
\hline Previously problematic & $5(4.1 \%)$ & $2(3.4 \%)$ & \\
\hline Problematic & $3(2.4 \%)$ & $0(0.0 \%)$ & 0.28 \\
\hline Heredity & $32(22.4 \%)$ & $26(36.6 \%)$ & 0.043 \\
\hline Hypercholesterolemia & $59(41.3 \%)$ & $22(30.6 \%)$ & 0.17 \\
\hline Stress & $33(23.2 \%)$ & $22(30.1 \%)$ & 0.35 \\
\hline \multicolumn{4}{|l|}{ Cardiovascular diseases } \\
\hline Ischemic heart disease & $81(56.6 \%)$ & $31(42.5 \%)$ & 0.067 \\
\hline Primary valvular disease & $12(8.4 \%)$ & $5(6.8 \%)$ & 0.92 \\
\hline Cardiomyopathy & $46(32.2 \%)$ & $26(35.6 \%)$ & 0.72 \\
\hline Myocarditis & $3(2.1 \%)$ & $1(1.4 \%)$ & 1.00 \\
\hline Chronic persistent atrial fibrillation & $0(0.0 \%)$ & $0(0.0 \%)$ & 1.00 \\
\hline Paroxysmal atrial fibrillation & $0(0.0 \%)$ & $0(0.0 \%)$ & 1.00 \\
\hline Cardiac arrest & $10(7.0 \%)$ & $1(1.4 \%)$ & 0.13 \\
\hline VES/VT & $28(19.6 \%)$ & $14(19.2 \%)$ & 1.00 \\
\hline SVT & $3(2.1 \%)$ & $4(5.5 \%)$ & 0.35 \\
\hline Bradycardia & $14(9.8 \%)$ & $5(6.8 \%)$ & 0.65 \\
\hline \multicolumn{4}{|l|}{ Non-cardiovascular diseases } \\
\hline Mild/moderate pulmonary disease & $15(10.5 \%)$ & $6(8.2 \%)$ & 0.79 \\
\hline Severe pulmonary disease & $0(0.0 \%)$ & $3(4.1 \%)$ & 0.075 \\
\hline Asthma & $7(4.9 \%)$ & $4(5.5 \%)$ & 1.00 \\
\hline \multicolumn{4}{|l|}{ GFR (cat.) } \\
\hline GFR $<30 \mathrm{ml} / \mathrm{min}$ & $3(2.1 \%)$ & $2(2.8 \%)$ & \\
\hline GFR $30-60 \mathrm{ml} / \mathrm{min}$ & $53(37.3 \%)$ & $24(33.8 \%)$ & \\
\hline GFR $>60 \mathrm{ml} / \mathrm{min}$ & $86(60.6 \%)$ & $45(63.4 \%)$ & 0.79 \\
\hline Missing & 1 & 2 & \\
\hline Stroke without sequelae & $10(7.0 \%)$ & $2(2.7 \%)$ & 0.33 \\
\hline Stroke with sequelae & $5(3.5 \%)$ & $1(1.4 \%)$ & 0.68 \\
\hline
\end{tabular}


Table 3 (continued)

\begin{tabular}{|c|c|c|c|}
\hline Variable & $\leq 70 \mathrm{bpm}(n=143)$ & $>70$ bpm $(n=73)$ & $p$ value \\
\hline Depression & $14(9.8 \%)$ & $10(13.7 \%)$ & 0.52 \\
\hline Impotence & $23(23.5 \%)$ & $8(16.7 \%)$ & 0.47 \\
\hline Malignancy (active) & $3(2.1 \%)$ & $1(1.4 \%)$ & 1.00 \\
\hline Malignancy (stable) & $15(10.5 \%)$ & $8(11.0 \%)$ & 1.00 \\
\hline Malnutrition & $1(0.7 \%)$ & $2(2.7 \%)$ & 0.53 \\
\hline Liver failure & $0(0.0 \%)$ & $0(0.0 \%)$ & 1.00 \\
\hline Thyroid disease & $10(7.0 \%)$ & $2(2.7 \%)$ & 0.33 \\
\hline Gout & $21(14.7 \%)$ & $5(6.8 \%)$ & 0.14 \\
\hline Dementia & $0(0.0 \%)$ & $1(1.4 \%)$ & 0.68 \\
\hline Other important non-cardiovascular disease & $13(9.1 \%)$ & $8(11.0 \%)$ & 0.83 \\
\hline \multicolumn{4}{|l|}{ Current status } \\
\hline \multicolumn{4}{|l|}{ Breathlessness_-Likert scale } \\
\hline Never & $45(31.5 \%)$ & $10(13.7 \%)$ & \\
\hline Upstairs & $75(52.4 \%)$ & $51(69.9 \%)$ & \\
\hline On level ground & $20(14.0 \%)$ & $7(9.6 \%)$ & \\
\hline In the shower & $3(2.1 \%)$ & $2(2.7 \%)$ & \\
\hline When resting & $0(0.0 \%)$ & $3(4.1 \%)$ & 0.015 \\
\hline \multicolumn{4}{|l|}{ Tiredness-Likert scale } \\
\hline Never & $59(41.3 \%)$ & $20(27.4 \%)$ & \\
\hline Upstairs & $63(44.1 \%)$ & $43(58.9 \%)$ & \\
\hline On level ground & $15(10.5 \%)$ & $5(6.8 \%)$ & \\
\hline In the shower & $4(2.8 \%)$ & $1(1.4 \%)$ & \\
\hline When resting & $2(1.4 \%)$ & $4(5.5 \%)$ & 0.12 \\
\hline \multicolumn{4}{|l|}{ Chest pain—Likert scale } \\
\hline Never & $128(89.5 \%)$ & $71(97.3 \%)$ & \\
\hline Upstairs & $10(7.0 \%)$ & $2(2.7 \%)$ & \\
\hline On level ground & $2(1.4 \%)$ & $0(0.0 \%)$ & \\
\hline In the shower & $1(0.7 \%)$ & $0(0.0 \%)$ & \\
\hline When resting & $2(1.4 \%)$ & $0(0.0 \%)$ & 0.048 \\
\hline Sitting systolic blood pressure $(\mathrm{mmHg})$ & $\begin{array}{l}123.1(15.8) \\
120.0(85.0 ; 165.0) \\
n=122\end{array}$ & $\begin{array}{l}123.4(22.4) \\
122.0(54.0 ; 180.0) \\
n=60\end{array}$ & 0.94 \\
\hline Standing systolic blood pressure (mmHg) & $\begin{array}{l}120.6(17.6) \\
120.0(80.0 ; 165.0) \\
n=113\end{array}$ & $\begin{array}{l}121.8(22.5) \\
122.5(70.0 ; 180.0) \\
n=58\end{array}$ & 0.80 \\
\hline LBBB & $44(30.8 \%)$ & $19(26.0 \%)$ & 0.57 \\
\hline \multicolumn{4}{|l|}{ NYHA } \\
\hline I & $32(22.4 \%)$ & $10(13.7 \%)$ & \\
\hline II & $81(56.6 \%)$ & $44(60.3 \%)$ & \\
\hline III & $30(21.0 \%)$ & $19(26.0 \%)$ & 0.14 \\
\hline
\end{tabular}

rhythm, we analyzed the interaction with EF or BBs leading to the risk of a HR $>70 \mathrm{bpm}$. Low EF is a recognized factor linked to a HR >70 bpm. BBs are assumed to impact HR. Interaction analyses were performed between $\mathrm{EF}$ and BBs vs. baseline data that included demographics, medical history, and clinical and laboratory data (Table 5; Fig. 3). There was no significant interaction with BBs but significant interactions between EF and the following variables as explanatory factors of $\mathrm{HF}>70 \mathrm{bpm}$ were observed: psychological stress, VPC/VT, GFR, and systolic blood pressure. In patients who had no stress, no VPC/VT, lower GFR, and lower SBP ( $<100 \mathrm{mmHg}$ ), EF caused a lower risk for HR $>70 \mathrm{bpm}$, whereas in patients with stress and VPC/VT, higher GFR and higher SBP (>140 mmHg) EF did not affect HR.

\section{Discussions}

This study reports suboptimal HR control in stable patients with HFrEF in an outpatient clinical setting. We also report 
Table 4 Medications and physicians' opinion regarding a HR $\leq 70$ vs. $>70$ bpm in patients with sinus rhythm and EF $<40 \%$

\begin{tabular}{|c|c|c|c|}
\hline Variable & $\leq 70 \mathrm{bpm}(n=143)$ & $>70 \mathrm{bpm}(n=73)$ & $p$ value \\
\hline Beta-blockers & $138(96.5 \%)$ & $70(95.9 \%)$ & 1.00 \\
\hline \multicolumn{4}{|l|}{ Beta-blockers (name) } \\
\hline Atenolol & $1(0.7 \%)$ & $0(0.0 \%)$ & \\
\hline Bisoprolol & $53(37.1 \%)$ & $31(42.5 \%)$ & \\
\hline Carvedilol & $12(8.4 \%)$ & $2(2.7 \%)$ & \\
\hline Metoprolol & $72(50.3 \%)$ & $37(50.7 \%)$ & \\
\hline Not using & $5(3.5 \%)$ & $3(4.1 \%)$ & 0.51 \\
\hline \multicolumn{4}{|l|}{ Reasons for not using BBs } \\
\hline Low blood pressure & $0(0.0 \%)$ & $1(1.4 \%)$ & 0.68 \\
\hline Dizziness & $1(0.7 \%)$ & $1(1.4 \%)$ & 1.00 \\
\hline Raynaud/Claudio & $0(0.0 \%)$ & $1(1.4 \%)$ & 0.68 \\
\hline Pulmonary disease & $0(0.0 \%)$ & $0(0.0 \%)$ & 1.00 \\
\hline Fatigue & $0(0.0 \%)$ & $2(2.7 \%)$ & 0.23 \\
\hline Bradycardia & $5(3.5 \%)$ & $0(0.0 \%)$ & 0.25 \\
\hline Asthma & $0(0.0 \%)$ & $0(0.0 \%)$ & 1.00 \\
\hline Decompensation & $0(0.0 \%)$ & $0(0.0 \%)$ & 1.00 \\
\hline No indication & $0(0.0 \%)$ & $0(0.0 \%)$ & 1.00 \\
\hline Other & $1(0.7 \%)$ & $1(1.4 \%)$ & 1.00 \\
\hline \multicolumn{4}{|l|}{ BB dose reached } \\
\hline$\geq 50$ target dose $\mathrm{e}^{\mathrm{a}}$ & $99(72.8 \%)$ & $56(80.0 \%)$ & 0.34 \\
\hline Target dose $\mathrm{e}^{\mathrm{a}}$ & $52(38.2 \%)$ & $28(40.0 \%)$ & 0.92 \\
\hline$>$ Target dose $\mathrm{a}^{\mathrm{a}}$ & $2(1.5 \%)$ & $0(0.0 \%)$ & 0.87 \\
\hline The maximum tolerated dose (physician's opinion) & $129(93.5 \%)$ & $59(84.3 \%)$ & 0.066 \\
\hline \multicolumn{4}{|l|}{ Reasons for not achieving BB target dose } \\
\hline Low blood pressure & $32(23.2 \%)$ & $15(21.4 \%)$ & 0.92 \\
\hline Fatigue & $12(8.7 \%)$ & $8(11.4 \%)$ & 0.69 \\
\hline Dyspnea & $3(2.2 \%)$ & $0(0.0 \%)$ & 0.58 \\
\hline Dizziness & $11(8.0 \%)$ & $9(12.9 \%)$ & 0.38 \\
\hline Bradycardia & $30(21.7 \%)$ & $3(4.3 \%)$ & 0.0010 \\
\hline Other & $14(10.1 \%)$ & $10(14.3 \%)$ & 0.51 \\
\hline \multicolumn{4}{|l|}{$\mathrm{BB}$ tolerated (on treatment with $\mathrm{BB}$ ) } \\
\hline No report of side effects & $87(60.8 \%)$ & $44(60.3 \%)$ & 1.00 \\
\hline Nightmares as side effect & $5(3.5 \%)$ & $2(2.7 \%)$ & 1.00 \\
\hline Cold extremities as side effect & $16(11.2 \%)$ & $3(4.1 \%)$ & 0.13 \\
\hline Impotence as side effect & $16(11.2 \%)$ & $2(2.7 \%)$ & 0.049 \\
\hline Depression as side effect & $2(1.4 \%)$ & $5(6.8 \%)$ & 0.090 \\
\hline Tiredness as side effect & $26(18.2 \%)$ & $17(23.3 \%)$ & 0.48 \\
\hline Other side effects & $3(2.1 \%)$ & $2(2.7 \%)$ & 1.00 \\
\hline \multicolumn{4}{|l|}{ BB up-titration done at } \\
\hline Department of Cardiology & $110(80.3 \%)$ & $59(83.1 \%)$ & \\
\hline Department of Medicine & $22(16.1 \%)$ & $11(15.5 \%)$ & \\
\hline Primary care & $5(3.6 \%)$ & $1(1.4 \%)$ & 0.65 \\
\hline BB duration (years) & $\begin{array}{l}3.60(4.55) \\
1.50(0.00 ; 19.80) \\
n=138\end{array}$ & $\begin{array}{l}3.01(4.31) \\
1.30(0.00 ; 18.10) \\
n=69\end{array}$ & 0.055 \\
\hline RAAS & $140(97.9 \%)$ & $69(94.5 \%)$ & 0.35 \\
\hline ACE inhibitors & $92(64.3 \%)$ & $44(60.3 \%)$ & 0.66 \\
\hline ARB & $51(35.7 \%)$ & $26(35.6 \%)$ & 1.00 \\
\hline \multicolumn{4}{|l|}{ ACE inhibitors (name) } \\
\hline Enalapril & $24(16.8 \%)$ & $19(26.0 \%)$ & \\
\hline
\end{tabular}


Table 4 (continued)

\begin{tabular}{|c|c|c|c|}
\hline Variable & $\leq 70 \mathrm{bpm}(n=143)$ & $>70 \operatorname{bpm}(n=73)$ & $p$ value \\
\hline Lisinopril & $0(0.0 \%)$ & $1(1.4 \%)$ & \\
\hline Not using & $51(35.7 \%)$ & $29(39.7 \%)$ & \\
\hline Ramipril & $68(47.6 \%)$ & $24(32.9 \%)$ & 0.082 \\
\hline \multicolumn{4}{|l|}{$\mathrm{ARB}$ (name) } \\
\hline Candesartan & $38(26.6 \%)$ & $18(24.7 \%)$ & \\
\hline Irbesartan & $0(0.0 \%)$ & $2(2.7 \%)$ & \\
\hline Losartan & $11(7.7 \%)$ & $5(6.8 \%)$ & \\
\hline Not using A & $92(64.3 \%)$ & $47(64.4 \%)$ & \\
\hline Valsartan & $2(1.4 \%)$ & $1(1.4 \%)$ & 0.40 \\
\hline ACE reached the maximum tolerated dose (physician's opinion) & $86(93.5 \%)$ & $36(81.8 \%)$ & 0.080 \\
\hline ARB reached the maximum tolerated dose (physician's opinion) & $43(82.7 \%)$ & $23(82.1 \%)$ & 1.00 \\
\hline RAAS reached the maximum tolerated dose (physician's opinion) & $125(89.3 \%)$ & $56(81.2 \%)$ & 0.16 \\
\hline MRA & $84(58.7 \%)$ & $42(57.5 \%)$ & 0.98 \\
\hline MRA reached the maximum tolerated dose (physician's opinion) & $74(88.1 \%)$ & $39(92.9 \%)$ & 0.62 \\
\hline \multicolumn{4}{|l|}{ Other treatments } \\
\hline Loop diuretics & $79(55.2 \%)$ & $40(54.8 \%)$ & 1.00 \\
\hline Digitalis & $4(2.8 \%)$ & $3(4.1 \%)$ & 0.88 \\
\hline Statin & $94(65.7 \%)$ & $42(57.5 \%)$ & 0.30 \\
\hline Nitrate & $17(11.9 \%)$ & $7(9.6 \%)$ & 0.79 \\
\hline Other thrombin inhibitors & $26(18.2 \%)$ & $16(21.9 \%)$ & 0.63 \\
\hline ASA & $78(54.5 \%)$ & $38(52.1 \%)$ & 0.84 \\
\hline Anticoagulants & $25(17.5 \%)$ & $12(16.4 \%)$ & 1.00 \\
\hline Antiarrhythmics other than BB & $3(2.1 \%)$ & $2(2.7 \%)$ & 1.00 \\
\hline Ivabradine/procoralan & $3(2.1 \%)$ & $6(8.2 \%)$ & 0.084 \\
\hline Allopur/probenecid & $19(13.3 \%)$ & $3(4.1 \%)$ & 0.051 \\
\hline \multicolumn{4}{|l|}{ Device treatments } \\
\hline Conventional pacemaker & $3(2.1 \%)$ & $6(8.2 \%)$ & 0.084 \\
\hline CRT & $20(14.0 \%)$ & $9(12.3 \%)$ & 0.91 \\
\hline ICD & $28(19.6 \%)$ & $18(24.7 \%)$ & 0.49 \\
\hline \multicolumn{4}{|l|}{ Clinical assessment } \\
\hline Physician considers patient having too low heart rate & $4(2.8 \%)$ & $1(1.4 \%)$ & 0.90 \\
\hline Physician considers patient being optimally treated & $129(90.2 \%)$ & $36(49.3 \%)$ & $<0.0001$ \\
\hline Physician considers patient having too high heart rate & $10(7.0 \%)$ & $36(49.3 \%)$ & $<0.0001$ \\
\hline
\end{tabular}

${ }^{\text {a }}$ Target dose is calculated only for patients using metoprolol (target $=200 \mathrm{mg}$ ), carvedilol (target $=50 \mathrm{mg}$ ), and bisoprolol (target $=10 \mathrm{mg}$ )

the distribution of HR in different categories of HF: HFrEF, HFmrEF, and HFpEF, both in sinus rhythm and AF, which, to our knowledge, has not been previously reported.

The mean HR of the HFrEF patients in sinus rhythm was $70 \mathrm{bpm}$ with $34 \%$ having $>70 \mathrm{bpm}$. This rate was lower than in our previous study (SwedeHF) in which about $47 \%$ of the patients had a HR $>70 \mathrm{bpm}$ [14]. However, there are several differences: first, the present study was a prospective investigation with a specific aim to study HR and, therefore, ECG was required to register HR at the time of inclusion; in SwedeHF the time point for HR could vary. Second, in the present study all HF patients were stable and in an outpatient clinical setting, whereas most of the patients in SwedeHF were hospitalized. However, the data from our current study were similar to another prospective multicenter study of patients with HFrEF and sinus rhythm in which $32 \%$ of the patients had HFs $\geq 70 \mathrm{bpm} \mathrm{[10].}$

\section{Possible causes for suboptimal target heart rate in HFrEF and sinus rhythm}

Two reasonable questions to ask are: why does HR differ across different studies and why does a HR of $>70 \mathrm{bpm}$ still occur in at least one-third of the HFrEF patients? As demonstrated in our study, non-achievement of the recommended target HR was unrelated to age, sex, cardiovascular risk factors, cardiovascular diseases, and comorbidities, but was related to EF and the clinical decision of the responsible physician. From our present and previous 
Table 5 Interaction analyses between LVEF (\%) and betablockers vs. demographics and clinical and laboratory data in an explanatory analysis of HR $\leq 70$ vs. $>70 \mathrm{bpm}$ in all patients with sinus rhythm and $\mathrm{EF}<40 \%$

\begin{tabular}{|c|c|c|}
\hline Interaction tested with variable & $\begin{array}{l}p \text { value for interaction with } \\
\text { LVEF }\end{array}$ & $\begin{array}{l}p \text { value for inter- } \\
\text { action with } \mathrm{BB}\end{array}$ \\
\hline Age (years) & 0.81 & 0.55 \\
\hline Sex & 0.19 & 0.96 \\
\hline NT-pro-BNP (ng/L) & 0.12 & 0.57 \\
\hline Hemoglobin $(\mathrm{g} / \mathrm{L})$ & 0.30 & 0.89 \\
\hline $\begin{array}{l}\text { Number of hospitalizations due to heart failure the past } \\
2 \text { years }\end{array}$ & 0.90 & 0.83 \\
\hline Hypertension & 0.20 & 1.00 \\
\hline $\mathrm{BMI}>30 \mathrm{~kg} / \mathrm{m}^{2}$ & 0.76 & 0.95 \\
\hline Smoking & 0.21 & 0.88 \\
\hline Diabetes & 0.62 & 0.95 \\
\hline Heredity & 0.15 & 0.95 \\
\hline Hypercholesterolemia & 0.96 & 0.29 \\
\hline Ischemic heart disease & 0.91 & 0.26 \\
\hline Primary valvular disease & 0.69 & 0.97 \\
\hline Cardiomyopathy & 0.38 & 0.23 \\
\hline Cardiac arrest & 0.42 & \\
\hline VES/VT & 0.018 & \\
\hline SVT & 0.90 & \\
\hline Bradycardia & 0.50 & 0.92 \\
\hline Mild/moderate pulmonary disease & 0.49 & 0.97 \\
\hline Severe pulmonary disease & 1.00 & \\
\hline Asthma & 0.18 & \\
\hline GFR (cat.) & 0.062 & 0.89 \\
\hline Stroke without sequelae & 0.29 & 0.97 \\
\hline Stroke with sequelae & 0.35 & \\
\hline Depression & 0.12 & 0.97 \\
\hline Impotence & 0.17 & 0.95 \\
\hline Malignancy (active) & 0.96 & \\
\hline Malignancy (stable) & 0.46 & 0.97 \\
\hline Thyroid disease & 0.26 & 0.98 \\
\hline Sitting systolic blood pressure (mmHg) & 0.14 & 0.76 \\
\hline Sitting systolic blood pressure (cat.) & 0.100 & 0.93 \\
\hline Standing systolic blood pressure $(\mathrm{mmHg})$ & 0.37 & 0.44 \\
\hline Standing systolic blood pressure (cat.) & 0.63 & 0.49 \\
\hline LBBB & 0.37 & 0.97 \\
\hline Chamber pacing & 0.45 & 0.95 \\
\hline NYHA & 0.44 & 0.27 \\
\hline Married/partner & 0.62 & 0.93 \\
\hline Working & 0.84 & 0.95 \\
\hline Retired & 0.88 & 0.95 \\
\hline
\end{tabular}

study [14], it appears that EF has an important impact on HR (i.e., lower EF is associated with higher HR), possibly implying that left ventricular function is one of the essential driving factors for higher HR.

Clinical assessment by physicians has received increased attention related to their roles in optimizing HF care [10-13], reflecting the awareness of and adherence to guideline-recommended treatment goals. In our study almost half of the physicians regarded a HR $>70 \mathrm{bpm}$ as optimal in HFrEF and sinus rhythm though equally many physicians considered a HR $>70 \mathrm{bpm}$ as being too high but without any plan for immediate action.

\section{Role of BBs for suboptimal target HR in HFrEF and sinus rhythm}

While the question of how BBs favorably influence the course of HF still remains unanswered, lowering HR is 


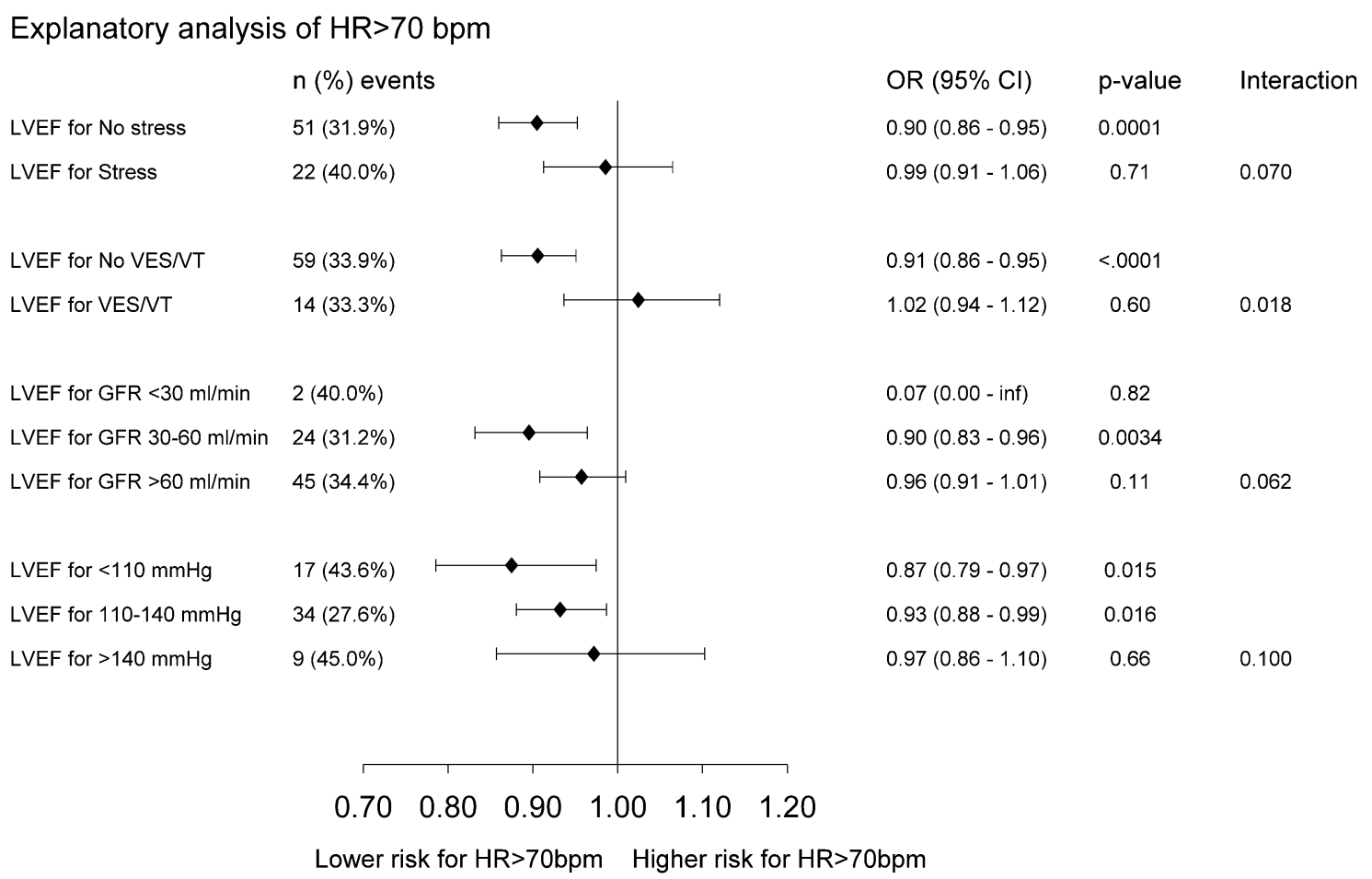

Fig. 3 Subgroup analysis of the effect of LVEF on HR in patients with sinus rhythm

considered very important [18, 19]. Although an increasing number of studies have demonstrated that a substantial proportion of patients with HFrEF does not tolerate the target doses of BBs used in large clinical trials [7, 10, 14, 20], dose issues surrounding BB appear persistent: first, when could we be certain that patients have reached the highest tolerable dose despite being below target dose? Second, how long should dose up-titration continue until it is certain that patients have reached the highest dose tolerable? As long as these questions remain unanswered, the addition of HRreducing therapies (such as ivabradine) will be postponed or questioned. Moran et al. argued that a lower use of BBs accounted for the difference between those attaining and those not attaining target HRs in stable HFrEF and sinus rhythm [10]. However, these findings could not be confirmed in our study. We did not observe any differences in the use of BBs between patients that had $<70 \mathrm{bpm}$ and those that had $>70 \mathrm{bpm}$, nor was there any interaction with BBs in patients with a HR $>70 \mathrm{bpm}$. Both prescription $(96 \%)$ and achieved target doses (40\%) of BBs were higher in our study than in the above-mentioned study (prescription $89 \%$ and achieved target doses 25\%) [10]. Taken together, these studies seem to suggest that despite differences in the use of BBs, a sizable proportion (approximately one-third) of the patients with HR $>70 \mathrm{bpm}$ was similar, suggesting that use of BBs is not the only explanation. Indeed, the proportion of $\mathrm{HR}$ $>70 \mathrm{bpm}$ is unrelated to the use of BBs as long as the BBs were up-titrated to the highest dose tolerable, which differs individually. As previously shown from the MERIT-HF trial, sicker patients did not tolerate higher doses of BBs, and despite this, the BBs were still effective, suggesting that it is the highest dose tolerable to patients that is all-important [20]. Further, as suggested from a recent meta-analysis, BB efficacy was significant in sinus rhythm, but not in AF, even though both groups showed a reduction in HR [21].

\section{Limitations}

The HF population enrolled in the study may not necessarily reflect the overall HF population. However, similar clinical characteristics in our study as compared with those from SwedeHF suggest the representativeness of our study population. Although participating investigators were encouraged to include patients consecutively we were unable to check that consecutive sampling was conducted.

\section{Implications}

Our data, together with available data $[6-10,14]$, underline that about one-third of the patients with HFrEF and sinus rhythm did not reach the target HR of $<70 \mathrm{bpm}$ as recommended by HF guidelines. However, this cannot be 
attributed to the use of BBs as long as they are administered in the highest tolerable dose. Further, approximately two-thirds of these patients will not tolerate the target dose, which actually has never been confirmed in a real-world setting.

A possible reason why physicians chose not to add ivabradine when the HR was $>70 \mathrm{bpm}$ might be that the recommendations from the EMA and most national pharmaceutical agencies are that ivabradine had an accepted indication if HR is $>75 \mathrm{bpm}$ [15-17]. The reason for this discrepancy is that survival benefit was shown in the SHIFT study in a subgroup with a heart rate of $75 \mathrm{bpm}$ or higher [22]. Several observational studies have found an association between elevated HR and poor survival. Our study indicates that among patients with $\mathrm{HFrEF}$, who were in sinus rhythm and on highest tolerable doses of betablockers, $14.3 \%$ might be eligible for ivabradine, which was similar to a previous study [9].

\section{Conclusion}

In this prospective survey of patients with stable HF in an outpatient clinical setting, we observed suboptimal HR control in HFrEF with sinus rhythm that was unrelated to the use of BBs. Our results support the position that concerted efforts and greater attention to control of HR in patients with HFrEF and sinus rhythm are needed.

Acknowledgements The study was initiated, organized, and monitored by the present investigators. We thank Aldina Pivodic, Statistiska Konsultgruppen, Gothenburg, Sweden, for conducting the statistical analysis. The study was supported by Servier Sweden AB.

Study organization: Principal Investigators: Michael Fu, professor, Section of Cardiology, Department of Medicine, Östra Hospital, Sahlgrenska University Hospital, Gothenburg, Sweden Bert Andersson, professor, Department of Cardiology, Sahlgrenska Hospital, Sahlgrenska University Hospital, Gothenburg, Sweden. Monitor: Sven Eric Hagelind, Research Unit, Department of Medicine, Östra Hospital, Sahlgrenska University Hospital, Gothenburg, Sweden. Participating centers (random order): Stefan Berglund, Falu Hospital; John-Erik Frisell, Ludvika Hospital; Bertil Borgencrantz, Capio Örebro; Agneta Månsson Broberg, Karolinska Hospital, Solna; Ulla Wedén, Karolinska Hospital, Huddinge; Carl Thorsén, Thoraxcenter, Blekinge Hospital, Karlshamn; Fredrik Kymle, Medicinenheten, Landskrona Hospital, Landskrona; Gordana Tasevska, Hjärtsvikt-och klaffsektionen, Skåne University Hospital/Malmö, Malmö; Anders Kullberg, Hjärthuset Varberg; Ulf Ahremark, Medicinkliniken Halmstad Hospital, Halmstad; Lars Andersson, Medicinkliniken Alingsås Hospital, Alingsås; Anders Ågård, Angereds Närsjukhus, Gothenburg; Anette Lehto, NÄL Hospital, Trollhättan; Anette Lehto, NÄL Hospital, Uddevalla; Bert Andersson, Sahlgrenska University Hospital/Sahlgrenska, Gothenburg; Michael Fu, Sahlgrenska University Hospital/Östra, Gothenburg; Niels Wagner, Södra Älvsborg Hospital, Borås; Gerhard Wikström, Akademiska University Hospital, Uppsala; Magnus Ehrsson, Karlstad Central Hospital, Karlstad; Julio Loayza, Karlskoga Hospital, Karlskoga; Carl-Johan Lindholm, Hjärtmottagning, Capio Citykliniken, Lund; Erasmus Bachus, Medicinkliniken, Hospital Ystad, Ystad.

\section{Compliance with ethical standards}

Disclosure Michael Fu and Bert Andersson report personal lecture fees from Servier and Novartis outside the submitted work. Other authors had nothing to disclose.

Open Access This article is distributed under the terms of the Creative Commons Attribution 4.0 International License (http://creativecommons.org/licenses/by/4.0/), which permits unrestricted use, distribution, and reproduction in any medium, provided you give appropriate credit to the original author(s) and the source, provide a link to the Creative Commons license, and indicate if changes were made.

\section{References}

1. McMurray JJ, Adamopoulos S, Anker SD, Auricchio A, Bohm M, Dickstein K, Falk V, Filippatos G, Fonseca C, Gomez-Sanchez MA, Jaarsma T, Kober L, Lip GY, Maggioni AP, Parkhomenko A, Pieske BM, Popescu BA, Ronnevik PK, Rutten FH, Schwitter J, Seferovic P, Stepinska J, Trindade PT, Voors AA, Zannad F, Zeiher A, Task Force for the Diagnosis and Treatment of Acute and Chronic Heart Failure 2012 of the European Society of Cardiology, Bax JJ, Baumgartner H, Ceconi C, Dean V, Deaton C, Fagard R, Funck-Brentano C, Hasdai D, Hoes A, Kirchhof P, Knuuti J, Kolh P, McDonagh T, Moulin C, Popescu BA, Reiner Z, Sechtem U, Sirnes PA, Tendera M, Torbicki A, Vahanian A, Windecker S, McDonagh T, Sechtem U, Bonet LA, Avraamides P, Ben Lamin HA, Brignole M, Coca A, Cowburn P, Dargie H, Elliott P, Flachskampf FA, Guida GF, Hardman S, Iung B, Merkely B, Mueller C, Nanas JN, Nielsen OW, Orn S, Parissis JT, Ponikowski P, ESC Committee for Practice Guidelines (2012) ESC guidelines for the diagnosis and treatment of acute and chronic heart failure 2012: the Task Force for the Diagnosis and Treatment of Acute and Chronic Heart Failure 2012 of the European Society of Cardiology. Developed in collaboration with the Heart Failure Association (HFA) of the ESC. Eur J Heart Fail 14:803-869

2. Yancy CW, Jessup M, Bozkurt B, Butler J, Casey DE Jr, Colvin MM, Drazner MH, Filippatos G, Fonarow GC, Givertz MM, Hollenberg SM, Lindenfeld J, Masoudi FA, McBride PE, Peterson PN, Stevenson LW, Westlake C (2016) 2016 ACC/AHA/HFSA Focused Update on New Pharmacological Therapy for Heart Failure: an update of the 2013 ACCF/AHA guideline for the management of heart failure: a report of the American College of Cardiology/American Heart Association Task Force on Clinical Practice Guidelines and the Heart Failure Society of America. J Am Coll Cardiol 68(13):1476-1488

3. Ponikowski P, Voors AA, Anker SD, Bueno H, Cleland JG, Coats AJ, Falk V, González-Juanatey JR, Harjola VP, Jankowska EA, Jessup M, Linde C, Nihoyannopoulos P, Parissis JT, Pieske B, Riley JP, Rosano GM, Ruilope LM, Ruschitzka F, Rutten FH, van der Meer P, Authors/Task Force Members (2016) 2016 ESC guidelines for the diagnosis and treatment of acute and chronic heart failure: the task force for the diagnosis and treatment of acute and chronic heart failure of the European Society of Cardiology (ESC) developed with the special contribution of the Heart Failure Association (HFA) of the ESC. Eur Heart J 37(27):2129-2200

4. Komajda M, Follath F, Swedberg K, Cleland J, Aguilar JC, CohenSolal A, Dietz R, Gavazzi A, Van Gilst WH, Hobbs R, Korewicki J, Madeira HC, Moiseyev VS, Preda I, Widimsky J, Freemantle N, Eastaugh J, Mason J (2003) Study Group of Diagnosis of the Working Group on Heart Failure of the European Society of 
Cardiology. The EuroHeart Failure Survey programme-a survey on the quality of care among patients with heart failure in Europe. Eur Heart J 24:464-474

5. de Groote P, Isnard R, Assyag P, Clerson P, Ducardonnet A, Galinier M, Jondeau G, Leurs I, Thebaut JF, Komajda M (2007) Is the gap between guidelines and clinical practice in heart failure treatment being filled? Insights from the IMPACT RECO survey. Eur J Heart Fail 9:1205-1211

6. Lenzen MJ, Boersma E, Scholteop Reimer WJM, Balk AHMM, Komajda M, Swedberg K, Follath F, Jimenez-Navarro M, Simoons ML, Cleland JGF (2005) Under-utilization of evidencebased drug treatment in patients with heart failure is only partially explained by dissimilarity to patients enrolled in landmark trials: a report from the Euro Heart Survey on Heart Failure. Eur Heart J 26:2706-2713

7. Maggioni AP, Anker SD, Dahlström U, Filippatos G, Ponikowski P, Zannad F, Amir O, Chioncel O, Crespo Leiro M, Drozdz J, Erglis A, Fazlibegovic E, Fonseca C, Fruhwald F, Gatzov P, Goncalvesova E, Hassanein M, Hradec J, Kavoliuniene A, Lainscak M, Logeart D, Merkely B, Metra M, Persson H, Seferovic $\mathrm{P}$, Termizhan A, Toussoulis D, Tavazzi L, on behalf of the Heart Failure Association of the ESC (HFA) (2013) Are hospitalized or ambulatory patients with heart failure treated in accordance with European Society of Cardiology guidelines? Evidence from 12.440 patients of the ESC Heart Failure Long-Term Registry. Eur J Heart Fail 15:1173-1184

8. Nieminen MS, Brutsaert D, Dickstein K, Drexler H, Follath F, Harjola CP, Hochadel M, Komajda M, Lassus J, Lopez-Sendon JL, Ponikowski P, Tavazzi L, EuroHeart Survey Investigators, Heart Failure Association, European Society of Cardiology (2006) EuroHeart Failure Survey II (EHFS II): a survey on hospitalized acute heart failure patients: description of population. Eur Heart J 27:2725-2736

9. Dierckx R, Cleland JG, Parsons S, Putzu P, Pellicori P, Dicken B, Boyalla V, Clark AL (2015) Prescribing patterns to optimize heart rate: analysis of 1,000 consecutive outpatient appointments to a single heart failure clinic over a 6-month period. JACC Heart Fail 3(3):224-230

10. Moran D, Buckley A, Daly K, Meaney B, Curtin R, O’Neill JO, Colwell N, Mahon N, Murphy N, O'Hanlon R, Daly C, Mc Adam B, Mc Donald K, Maher V (2014) Heart rate awareness in patients with chronic stable heart failure. A multi-center observational study. Int J Cardiol 177(2):380-384

11. Gjesing A, Schou M, Torp-Pedersen C, Kober L, Gustafsson F, Hildebrandt P, Videbaek L, Wiggrs H, Demand M, Charlot M, Gislason GH (2013) Patient adherence to evidence-based pharmacotherapy in systolic heart failure and the transition of follow-up from specialized heart failure outpatient clinics to primary care. Eur J Heart Fail 15:671-678

12. Poelzl G, Altenberger J, Pacher R, Ebner CH, Wieser M, Winter A, Fruhwald F, Dornaus C, Ehmsen U, Reiter S, Steinacher R,
Huelsmann M, Eder V, Boehmer A, Pilgersdorfer L, Ablasser K, Keroe D, Groebner H, Auer J, Jakl G, Hallas A, Ess M, Ulmer $\mathrm{H}$ (2014) Dose matters! Optimisation of guideline adherence is associated with lower mortality in stable patients with chronic heart failure. Int J Cardiol 175:83-89

13. Fonarow GC, Yancy CW, Albert NM, Curtis AB, Gattis Stough W, Gheorghiade M, Heywood T, McBride ML, Mehra MR, O'Connor CM, Reynolds D, Walsh MN (2008) Heart failure care in the outpatient cardiology practice setting. Findings from IMPROVE HF. Circ Heart Fail 1:98-106

14. Li SJ, Sartipy U, Lund LH, Dahlström U, Adiels M, Petzold M, $\mathrm{Fu}$ M. Prognostic significance of resting heart rate and use of $\beta$-blockers in atrial fibrillation and sinus rhythm in patients with heart failure and reduced ejection fraction: findings from the Swedish Heart Failure Registry. Annual report 2015 http://www. ucr.uu.se/rikssvikt/index.php/om-rikssvikt/arsrapporter

15. Böhm M, Swedberg K, Komajda M, Borer JS, Ford I, DubostBrama A, Lerebours G, Tavazzi L, SHIFT Investigators (2010) Heart rate as a risk factor in chronic heart failure (SHIFT): the association between heart rate and outcomes in a randomised placebo-controlled trial. Lancet 376(9744):886-894

16. Swedberg K, Komajda M, Bohm M et al (2012) Effects on outcomes of heart rate reduction by ivabradine in patients with congestive heart failure: is there an influence of beta-blocker dose? Findings from the SHIFT (systolic Heart failure treatment with the I(f) inhibitor ivabradine Trial) study. J Am Coll Cardiol 59(22):1938-1945

17. Borer JS, Bohm M, Ford I et al (2012) Effect of ivabradine on recurrent hospitalization for worsening heart failure in patients with chronic systolic heart failure: the SHIFT study. Eur Heart J 33(22):2813-2820

18. Sarraf M, Francis GS (2012) It is all about heart rate. Or is it? Am. J Am Coll Cardiol 59(22):1946-1947

19. Maurer MS, Sackner-Bernstein JD, El-Khoury Rumbarger L, Yushak M, King DL, Burkhoff D (2009) Mechanisms underlying improvements in ejection fraction with carvedilol in heart failure. Circ Heart Fail 2:189-196

20. Wikstrand J, Hjalmarson A, Waagstein F, Fagerberg B, Goldstein $\mathrm{S}$, Kjekshus J et al (2002) Dose of metoprolol CR/XL and clinical outcomes in patients with heart failure: analysis of the experience in metoprolol $\mathrm{CR} / \mathrm{XL}$ randomized intervention trial in chronic heart failure (MERIT-HF). J Am Coll Cardiol 40(3):491-498

21. Kotecha D, Holmes J, Krum H, Altman DG, Manzano L, Cleland JG et al (2014) Efficacy of beta blockers in patients with heart failure plus atrial fibrillation: an individual-patient data metaanalysis. Lancet 384(9961):2235-2243

22. Böhm M, Borer J, Ford I, Gonzalez-Juanatey JR, Komajda M, Lopez-Sendon J, Reil JC, Swedberg K, Tavazzi L (2013) Heart rate at baseline influences the effect of ivabradine on cardiovascular outcomes in chronic heart failure: analysis from the SHIFT study. Clin Res Cardiol 102(1):11-22 